\title{
Minimal model of charge and pairing density waves in x-ray scattering experiments
}

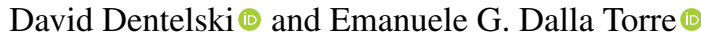 \\ Department of Physics, Bar-Ilan University, 52900 Ramat Gan, Israel \\ and Center for Quantum Entanglement Science and Technology, Bar-Ilan University, 52900 Ramat Gan, Israel
}

(Received 13 August 2019; revised 17 December 2019; accepted 22 June 2020; published 10 July 2020)

\begin{abstract}
Competing density waves play an important role in the mystery of high-temperature cuprate superconductors. In spite of the large amount of experimental evidence, the fundamental question of whether these modulations represent charge or pairing density waves (CDWs or PDWs) is still debated. Here we present a method to answer this question using both momentum and energy-resolved resonant $\mathrm{x}$-ray-scattering maps. Starting from a minimal model of density waves in superconductors, we identify distinctive signatures of incipient CDWs and PDWs. The generality of our approach is confirmed by a self-consistent solution of an extended Hubbard model with attractive interaction. By considering the available experimental data, we claim that the spatial modulations in cuprates have a predominant PDW character. Our work paves the way for using $\mathrm{x}$ ray to identify competing and intertwined orders in superconducting materials.
\end{abstract}

DOI: 10.1103/PhysRevResearch.2.032012

Introduction. Strongly correlated materials often exhibit competing phases with distinct charge and spin orders. A famous example is copper-oxide high-temperature superconductors, or cuprates, whose rich phase diagram poses many theoretical challenges. Since the discovery of unidirectional spin density waves in $\mathrm{La}_{2-x} \mathrm{Sr}_{x} \mathrm{CuO}_{4}$ (LSCO) [1], it has become increasingly accepted that in cuprates superconductivity is intertwined with other orders [2-6]. In particular, in 2002, scanning tunneling experiments found incommensurate density waves on the surface of $\mathrm{Bi}_{2} \mathrm{Sr}_{2} \mathrm{CaCu}_{2} \mathrm{O}_{8+x}$ (BSCCO) [7-10]. Ten years later, resonant $\mathrm{x}$-ray-scattering experiments detected a similar incommensurate order in the bulk of $\mathrm{YBa}_{2} \mathrm{Cu}_{3} \mathrm{O}_{7-x}$ (YBCO) [11]. The same order was later found in a large number of cuprates, demonstrating that this effect is ubiquitous [12-30].

In spite of the large number of experimental studies, the physical interpretation of these periodic modulations is still debated. A common approach, also based on earlier theoretical predictions [31,32], claims that these modulations are due to a charge density wave (CDW) order that competes with superconductivity. While this approach is widely accepted in the literature, it is inconsistent with some experimental details. In particular, angle-resolved photoemission spectroscopy (ARPES) shows that these density waves are associated with a spectral gap that closes from below the Fermi energy [33], while CDWs' gaps are expected to close from above. Accordingly, it was argued that the competing order is intimately related to superconductivity [34] and thus interpreted as a pair density wave (PDW) $[35,36]$, or a CDW/PDW mixed order [37-40]. This claim is also supported by recent

Published by the American Physical Society under the terms of the Creative Commons Attribution 4.0 International license. Further distribution of this work must maintain attribution to the author(s) and the published article's title, journal citation, and DOI. scanning measurements in the halos of magnetic vortices [41] and with superconducting tips [42,43] (see Ref. [44] for a recent review).

Here we address the question of how to distinguish between CDW and PDW modulations in available x-rayscattering experiments. Our approach departs from earlier studies that focused on the normal state of cuprates $[45,46]$ and included the effects of strong antiferromagnetic fluctuations [47] and Fermi arcs with hot spots [37-39,39,40,48,49]. Instead, we base our analysis on the well-established description of the superconducting state of cuprates in terms of a Bardeen-Cooper-Schrieffer (BCS) Hamiltonian with a $d$-wave gap. In this phase, superconductivity suppresses competing orders, justifying a weak-coupling approach where the density waves are induced by weak pinning centers [50,51]. By considering a minimal model of isotropic scatterers, we develop a method to distinguish between incipient CDW and PDW fluctuations, which become long-ranged at high magnetic fields. The validity of this approach is confirmed by the solution of an extended Hubbard model with attractive interactions in the presence of local impurities, which enables us to study the interplay between CDWs and PDWs.

Weak-coupling approach. Resonant x-ray-scattering experiments probe density fluctuations at a fixed wave vector $\mathbf{q}$ and frequency $\Omega$. In our weak-coupling approach we assume that incipient CDWs and PDWs can be modeled by a homogeneous state perturbed by a local pinning center (impurity). Under this approximation, the intensity of the x-ray signal is given by density response to the impurity, $\chi(\mathbf{q}, \Omega)$. In a BCS superconductor, one has (see, e.g., Ref. [52])

$$
\chi(\mathbf{q}, \Omega)=\int d \omega \int d^{d} k \operatorname{Tr}\left[G_{0}(\mathbf{k}, \omega) V_{\mathbf{k}} G_{0}(\mathbf{k}+\mathbf{q}, \omega+\Omega) \sigma^{z}\right] .
$$

Here $V$ models a static (time-independent) and local ( $q$ independent) impurity, $\sigma^{z}$ is a Pauli matrix, and $G_{0}$ is the 
(a) $\mathrm{NCCO}$

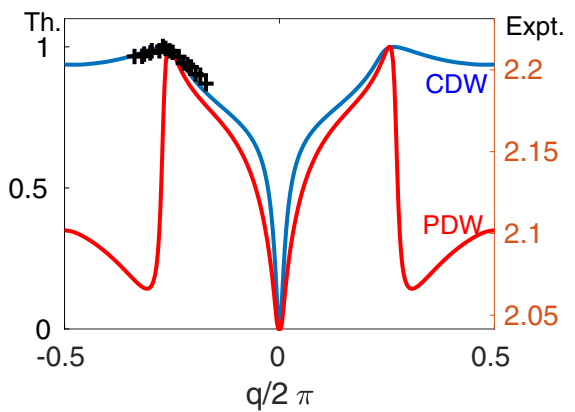

(b) $\operatorname{Hg} 1201$

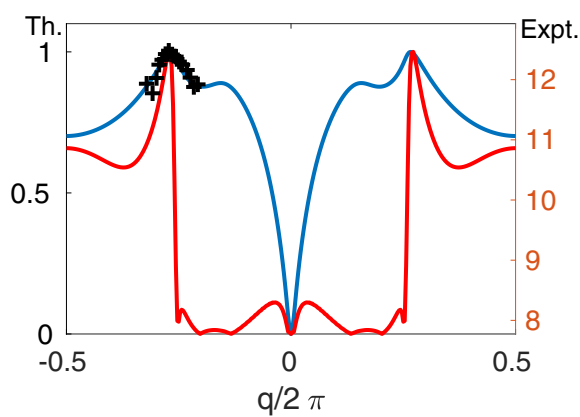

(c) BSCCO

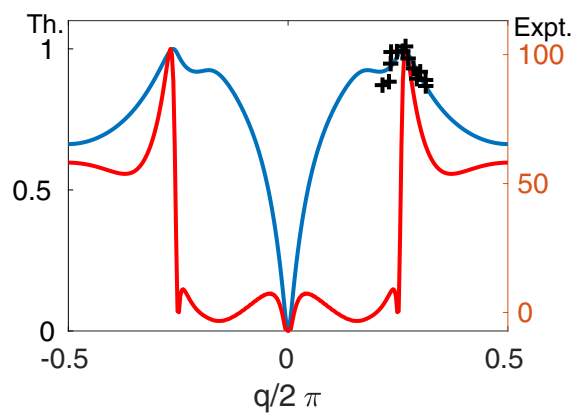

FIG. 1. Momentum dependence of the theoretical (Th.,- continuous lines) and experimental (Expt., crosses) elastic x-ray-scattering signal in the $(q, 0)$ direction. The theoretical curves of incipient CDWs (PDWs) are shown in blue (red) and represent Eq. (3) [Eq. (4)] with $\Omega=$ $0, \Delta_{0} / t=0.1\left(\Delta_{0} / t=0.3\right)$ and (a) $t^{\prime} / t=-0.22(-0.4)$, (b) $t^{\prime} / t=-0.7(-0.7)$, and (c) $t^{\prime} / t=-0.7(-0.7)$. Each theoretical curve is normalized by its maximal value. The experimental data is reproduced from (a) electron-doped NCCO, $x=-0.14$ [27]; (b) underdoped $\operatorname{Hg} 1201, x=0.09$ [19]; and (c) underdoped BSCCO, $x=0.12$ [15].

Green's function

$$
G_{0}^{-1}(\mathbf{k}, \omega)=\left(\begin{array}{cc}
-\omega+\varepsilon_{\mathbf{k}}-\mu & \Delta_{\mathbf{k}} \\
\Delta_{\mathbf{k}}^{\star} & -\omega-\varepsilon_{\mathbf{k}}+\mu
\end{array}\right),
$$

where $\Delta_{\mathbf{k}}=\frac{\Delta_{0}}{2}\left[\cos \left(k_{x}\right)-\cos \left(k_{y}\right)\right]$ is the pairing gap, $\varepsilon_{\mathbf{k}}$ is the band structure of the material, and $\mu$ the chemical potential [53].

We now introduce a minimal model for CDW modulations, by considering the scattering from a momentum-independent charge impurity, $V_{\mathbf{k}}=V_{0} \sigma^{z}$. In this case, the integral over $\omega$ in Eq. (1) delivers

$$
\begin{aligned}
\chi(\mathbf{q}, \Omega)= & 2 \pi V_{0} \int d^{2} k \frac{E_{\mathbf{k}}^{2}+\varepsilon_{\mathbf{k}} \varepsilon_{\mathbf{k}+\mathbf{q}}-\Delta_{\mathbf{k}} \Delta_{\mathbf{k}+\mathbf{q}}-E_{\mathbf{k}} \Omega}{E_{\mathbf{k}}\left[E_{\mathbf{k}+\mathbf{q}}^{2}-\left(E_{\mathbf{k}}-\Omega\right)^{2}\right]} \\
& +\frac{E_{\mathbf{k}+\mathbf{q}}^{2}+\varepsilon_{\mathbf{k}} \varepsilon_{\mathbf{k}+\mathbf{q}}-\Delta_{\mathbf{k}} \Delta_{\mathbf{k}+\mathbf{q}}+E_{\mathbf{k}+\mathbf{q}} \Omega}{E_{\mathbf{k}+\mathbf{q}}\left[E_{\mathbf{k}}^{2}-\left(E_{\mathbf{k}+\mathbf{q}}+\Omega\right)^{2}\right]},
\end{aligned}
$$

where $E_{\mathbf{k}}=\sqrt{\varepsilon_{\mathbf{k}}^{2}+\Delta_{\mathbf{k}}^{2}}$. In the limit of $\Delta_{\mathbf{k}} \rightarrow 0$, one has $E_{\mathbf{k}}=$ $\left|\varepsilon_{\mathbf{k}}\right|$ and Eq. (3) recovers the Lindhard response function of free fermions used in Ref. [45].

To describe $\mathrm{x}$-ray-scattering experiments of cuprates, we use a tight-binding model, $\varepsilon_{\mathbf{k}}=-2 t\left[\cos \left(k_{x}\right)+\cos \left(k_{y}\right)\right]-$ $4 t^{\prime} \cos \left(k_{x}\right) \cos \left(k_{y}\right)$, where $t$ and $t^{\prime}$ are nearest-neighbor (NN) and next-nearest-neighbor (NNN) hopping coefficients. The parameter $t^{\prime}$ strongly affects the shape of the Fermi surface: superconducting cuprates are close to half-filling and, for $t^{\prime}=0$, their Fermi surface has a diamond shape. A negative $t^{\prime}$ leads to a Fermi surface with parallel segments (nesting) at the antinodal wave vectors $\mathbf{k}=( \pm \pi / a, 0)$ and $(0, \pm \pi / a)$. As pointed out long ago [54], these parallel segments are prone to induce finite wave-vector instabilities, such as CDWs and PDWs. This approach matches the experimentally observed doping dependence of the wave vector, see the Supplemental Material [55].

Let us first consider the elastic component $(\Omega=0)$, by comparing Eq. (3) with resonant $\mathrm{x}$-ray-scattering experiments of three different cuprates: electron-doped $\mathrm{Nd}_{2-x} \mathrm{Ce}_{x} \mathrm{CuO}_{4}$ (NCCO) [27], underdoped BSCCO [15], and underdoped $\mathrm{HgBa}_{2} \mathrm{CuO}_{4+x}(\mathrm{Hg} 1201)$ [19]. The corresponding plots are shown as blue curves in Fig. 1, where we select $\mu$ to match the experimental doping $x$. The superconducting gap $\Delta_{0}$ has a minor influence on these plots and is set to physically relevant values. The fitting parameter $t^{\prime}$ is obtained by minimizing the difference between the theoretical curves and the actual experiments [56]. The values of $t^{\prime} / t$ obtained by this procedure are consistent with the Fermi surfaces determined by ARPES [57,58]. For all three materials, we obtain an excellent agreement between the theoretical curves and the experiments: Eq. (3) describes well both the period of the modulation and the width of the peak.

As mentioned above, an alternative explanation for the observed signal is PDW fluctuations. Specifically, we consider short-ranged PDWs that coexist with a static and uniform $(d-$ wave) pairing gap $\Delta_{0}$. Our analysis does not apply to materials where the PDWs are long ranged and give rise to a state, analogous to the Fulde-Ferrell-Larkin-Ovchinnikov (FFLO) state $[59,60]$, where the pairing gap is periodically modulated in space (such as the striped superconductor $\mathrm{La}_{2-x} \mathrm{Ba}_{x} \mathrm{CuO}_{4}$ (LBCO) near $1 / 8$ doping [61,62]). Following our weakcoupling approach, we consider PDW fluctuations induced by a local modulation of the pairing gap, $V_{\mathbf{k}}=\Delta_{\mathbf{k}} \sigma^{x}$ in Eq. (1), where $\sigma^{x}$ is a Pauli matrix. By performing the integral over $\omega$ in Eq. (1), we obtain

$$
\chi(\mathbf{q}, \Omega)=2 \pi \int d^{2} k \Delta_{\mathbf{k}} \frac{\varepsilon_{\mathbf{k}} \Delta_{\mathbf{k}+\mathbf{q}}+\varepsilon_{\mathbf{k}+\mathbf{q}} \Delta_{\mathbf{k}}}{\left(E_{\mathbf{k}}-E_{\mathbf{k}+\mathbf{q}}\right)^{2}-\Omega^{2}}\left(\frac{1}{E_{\mathbf{k}}}-\frac{1}{E_{\mathbf{k}+\mathbf{q}}}\right) .
$$

The resulting plots are shown as red curves in Fig. 1. We find that the PDW signal shows pronounced peaks at approximately the same wave vector as the CDW one. The precise shape of the peaks depends on the details of the band structure and cannot be used to identify the type of modulation. As a result, one-dimensional scans of the x-ray scattering are not sufficient to distinguish unequivocally between CDW and PDW fluctuations.

Identifying $C D W$ and $P D W$. We now present two distinct methods to distinguish between these two types of modulations, based respectively on the momentum and energy dependence of the x-ray-scattering signal. The first method uses the 
(a) CDW

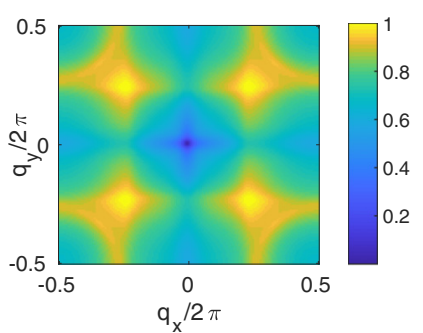

(b) PDW

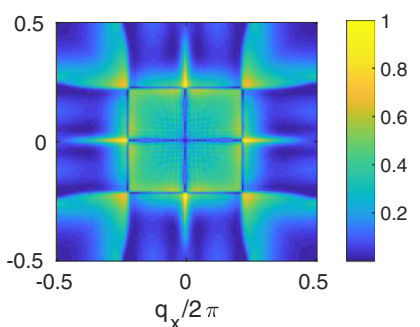

FIG. 2. Two dimensional maps of the elastic response $\chi(\mathbf{q}, \Omega=$ 0 ) in the weak-coupling approach: (a) CDW, Eq. (3), (b) PDW, Eq. (4). Numerical parameters: $x=0.14, t^{\prime} / t=-0.6, \Delta_{0} / t=0.2$.

full two-dimensional map of $\chi(\mathbf{q}, \Omega=0)$. Two representative theoretical maps are shown in Fig. 2. Although both maps have pronounced peaks at the same wave vector $(q \approx \pm 0.25)$, their two-dimensional structure is very different: The CDW signal has four peaks at $\mathbf{q}=( \pm q, \pm q)$ and four saddle points at $\mathbf{q}=(0, \pm q)$ and $( \pm q, 0)$. In contrast, the PDW signal has four strong peaks at $\mathbf{q}=(0, \pm q)$ and $( \pm q, 0)$ and four weaker peaks at $\mathbf{q}=( \pm q, \pm q)$. We claim that the ratio between the intensity of the signal at these two wave vectors can be used to identify the type of modulation. For the parameters used in Fig. 2, we find that $R \equiv \chi[\mathbf{q}=(q, 0), \Omega=0] / \chi(\mathbf{q}=$ $(q, q), \Omega=0) \approx 0.7$ for CDW and $R \approx 1.4$ for PDW.

This result is very robust: Although the precise value of $R$ depends on the microscopic parameters of the model, we find generically that $R<1$ for CDWs and $R>1$ for PDWs.

To understand this result, we recall that in our weakcoupling model of CDW and PDW, the scatterers are local and isotropic and, hence, their scattering matrices are momentum independent. In this minimal model, the intensity of the response function only depends on the density of states, i.e., on the shape of the Fermi surface and on the symmetry of the pairing gap. As mentioned before, the Fermi surface of cuprates has four pairs of parallel segments, leading to a doping-dependent nesting wave vector $q$. Interestingly, the nesting at wave vector $(q, q)$ is more effective than at wave vector $(q, 0)$ : In the former case one obtains an overlap between all four pairs of segments of the Fermi surface, while in the latter only two pairs are involved. This observation explains why the CDW is more pronounced at wave vector $(q, q)$ than at $(q, 0)$, i.e., $R<1$ [63]. In the case of the PDW signal, Eq. (4), each segment of the Fermi surface is weighted by the corresponding value of $\Delta_{\mathbf{k}}$. This factor strongly favors the wave vector $(q, 0)$, which connects antinodes to antinodes, with respect to $(q, q)$, which connects antinodes to nodes. Hence, for PDWs $R>1$ in agreement with the numerical result mentioned above.

The experimental data strongly supports the PDW scenario: (i) Transverse and longitudinal one-dimensional scans in the vicinity of $(q, 0)$ show that the scattering amplitude peaks in both directions [26]. This experimental finding is in stark contradiction with the CDW case, where a saddle point is expected, and agrees with the PDW case (see Fig. 2). (ii) The peak at $(q, q)$ is small [64] or absent [30,46], indicating that $R>1$, whereas for CDW this should be the dominant peak. Both observations are consistent with the PDW scenario only.

(a) CDW
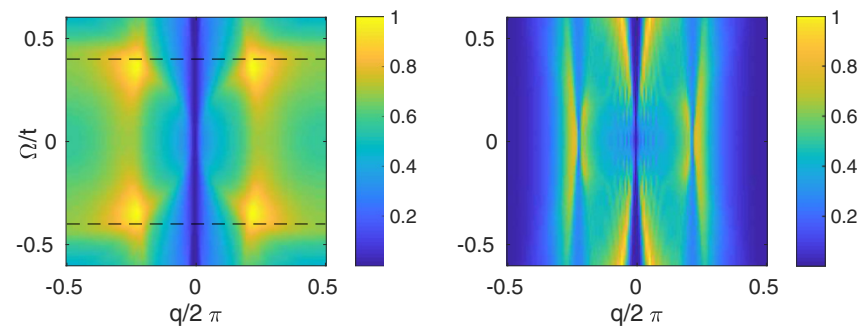

FIG. 3. Energy-momentum dependence of the inelastic response $\chi(\mathbf{q}, \Omega)$ along the line $(q, 0)$ in the weak-coupling approach for (a) CDWs, Eq. (3), and (b) PDWs, Eq. (4). Same numerical values as in Fig. 2. The black dashed lines in (a) are located at $\Omega / t= \pm 2 \Delta_{0} / t$.

Energy dependence. Let us now turn to the energy dependence of the response function $\chi(\mathbf{q}, \Omega)$. Figure 3 shows our theoretical predictions for incipient CDWs, Eq. (3), and PDWs, Eq. (4). The energy dependence of the two graphs is very different: $\chi(\mathbf{q}, \Omega)$ is peaked at $\Omega \approx 2 \Delta_{0}$ for CDWs and at $\Omega=0$ for PDWs. This discrepancy can be rationalized by noting that charge impurities create particle-hole pairs and, hence, need to overcome the energy gap $\Delta$. In contrast, local modulations of the pairing gap can create two holes (or two particles) at the same energy, below (or above) the gap. As a consequence, the response to CDWs is peaked at $2 \Delta_{0}$, while the response to PDWs is peaked at zero energy. Recent energy-resolved inelastic x-ray-scattering (RIXS) experiments $[11,25,26,28,46]$ show that the signal is peaked at (or close to) zero energy and, again, favor the PDW scenario. Furthermore, our theoretical model accounts for the experimental observation of a dispersive peak that departs from the zero-energy peak towards higher energies [25].

Hubbard model. The weak-coupling approach considered above does not take into account the interactions between quasiparticles, which can enhance the CDW and PDW fluctuations and lead to a competition between them. To capture these effects, we now consider a two-dimensional extended Hubbard model with on-site repulsion $U$ and $\mathrm{NN}$ attraction $V$. This model shows several competing phases, such as the Mott insulator and the $d$-wave superconductor, that are generic to cuprates [65-70]. Under the usual mean-field approximation $n_{j}=\sum_{\sigma= \pm}\left\langle c_{j, \sigma}^{\dagger} c_{j, \sigma}\right\rangle / 2, \Delta_{\hat{e}, j}=\sum_{\sigma= \pm} \sigma\left\langle c_{j,-\sigma} c_{j+\hat{e}, \sigma}\right\rangle / 2$, the Hamiltonian reads

$$
\begin{aligned}
H= & -t \sum_{\langle i, j\rangle, \sigma} c_{i, \sigma}^{\dagger} c_{j, \sigma}-t^{\prime} \sum_{\langle\langle i, j\rangle\rangle, \sigma}\left(c_{i, \sigma}^{\dagger} c_{j, \sigma}+\text { H.c. }\right) \\
& +U \sum_{j, \sigma} n_{j} c_{j, \sigma}^{\dagger} c_{j, \sigma}+V \sum_{\hat{e}, j, \sigma}\left(\Delta_{\hat{e}, j} c_{j, \uparrow}^{\dagger} c_{j+\hat{e}, \downarrow}^{\dagger}+\text { H.c. }\right),
\end{aligned}
$$

where $\langle\cdot, \cdot\rangle$ denotes NN, $\langle\langle\cdot, \cdot\rangle\rangle$ denotes NNN, $\hat{e}$ connects NN sites, and H.c. stands for Hermitian conjugate. For $V<0$, the self-consistent solution of Eq. (5) delivers a superconductor with $d$-wave order parameter $\Delta_{j}=\frac{1}{4}\left(\Delta_{\hat{x}, j}+\Delta_{-\hat{x}, j}-\Delta_{\hat{y}, j}-\right.$ $\left.\Delta_{-\hat{y}, j}\right)$.

To study the interplay between CDW and PDW we add a local impurity, which generically leads to spatial modulations of both the charge $n_{j}$ and the pairing gap $\Delta_{j}$. We classify the 
(a) site impurity $(\delta U)$

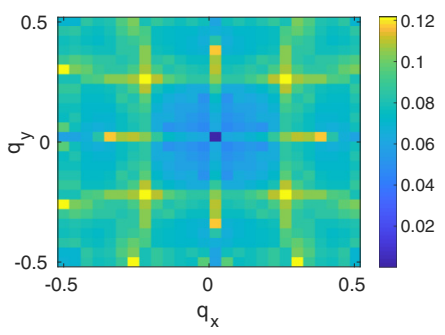

(b) bond impurity $(\delta V)$

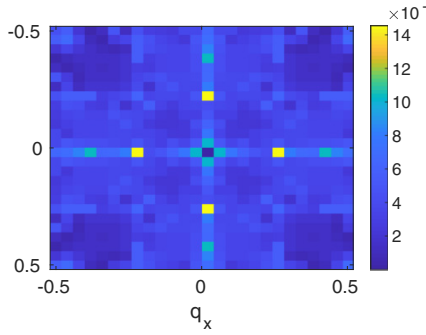

FIG. 4. Two-dimensional maps of the density fluctuations $n_{\mathbf{q}}$ in the Hubbard model with a local modulation of the interaction: (a) on a single site; (b) on a single bond. For clarity, (b) has been symmetrized by $90^{\circ}$. Numerical parameters: $x=0.16, t^{\prime} / t=-0.6$ and $U / t=1.5, V / t=-0.5, \delta U / t=\delta V / t=0.2$.

resulting density wave as CDW or PDW depending on which modulation is dominant, by comparing the relative standard deviations $\delta n$ and $\delta \Delta$ [71]. For simplicity, we focus here on two representative impurities (see the Supplemental Material for additional examples): a single site with $U \rightarrow U+\delta U$ and a single bond with $V \rightarrow V+\delta V$. In the former case, we find that the density wave has mixed CDW/PDW character $(\delta \Delta / \delta n \approx 1)$, while in the latter case it has a predominant $\operatorname{PDW}$ character $(\delta \Delta / \delta n \approx 140)$.

To model the response to $\mathrm{x}$-ray scattering, we now focus on the Fourier transformed density $n_{\mathbf{q}}$, Fig. 4 . As expected, we find that interactions enhance density wave instabilities and lead to narrower peaks, with longer range correlations. In our calculations, the width of these peaks is limited by the system size ( $L=26$ unit cells), suggesting that the Hubbard model is consistent with long-ranged CDW/PDW modulations. By comparing the two subplots, we observe that the on-site impurity leads to peaks at both wave vectors $(q, q)$ and $(q, 0)$, while the bond impurity leads to pronounced peaks in at the wave vector $(q, 0)$ only. This is consistent with our proposal to compare the intensities of the two peaks to distinguish between CDW and PDW modulations.

Summary and discussion. In this Rapid Communication we described a method to distinguish between incipient CDW and PDW fluctuations, based on the analysis of x-ray-scattering experiments. When these spatial modulations coexist with a homogeneous superconducting gap $\Delta_{0}$, both oscillations couple directly to any physical observable, such as the charge density and the tunneling density of states. Hence, both CDWs and PDWs can be detected using different experimental techniques, including $\mathrm{x}$-ray scattering, scanning tunneling spectroscopy (STS), and scanning Josephson probes. Here we focused on X-ray scattering and showed that one-dimensional cuts are not unequivocal, because they can be adequately fitted by both types of modulations. In contrast, two-dimensional maps of CDWs and PDWs are very different: the former are peaked at wave vector $(q, q)$, while the latter has stronger peaks at $(q, 0)$. These two types of density waves can be further distinguished by energy-resolved RIXS measurements: CDWs are peaked at $\Omega=2 \Delta_{0}$, while PDWs are peaked at $\Omega=0$.
These results strongly rely on our simplifying assumption of noninteracting quasiparticles, scattered by local and isotropic pinning centers. In principle, other sources of inhomogeneity, as well as strong electronic correlations, can yield different results and enhance CDW and PDW signals in other directions. To address this point, we considered an attractive Hubbard model, which demonstrated that our method to identify fluctuations with a dominant CDW or PDW character remains valid in the presence of strong interactions. In particular, we showed that local modulations of the pairing mechanism (in our case, a nearest-neighbor attractive interaction) give rise to density waves peaked at $(q, 0)$ and with a predominant PDW character.

Our theoretical model of PDW reproduces the main features of recent $\mathrm{x}$-ray-scattering experiments of superconducting NCCO, Hg1201, and BSCCO. Specifically, our theory explains why (i) the X-ray-scattering signal is peaked at wave vector $(q, 0)$, rather than at $(q, q)$, as expected for CDWs; (ii) the RIXS signal is peaked at frequency $\Omega=0$ and is accompanied by weaker dispersive inelastic peaks. Our findings also agree with earlier STS experiments of BSCCO [8-10], which found that the incommensurate checkerboard order has a dominant PDW character [72-75]. Attributing the x-ray signal to fluctuations of the pairing order parameter (PDW) explains its temperature dependence: these fluctuations are strongest at the critical temperature of superconducting order parameter, $T_{c}$, in agreement with the experimental observations ([12,16]). Finally, the proposed PDW scenario explains why the signal detected in X-ray scattering of cuprates is orders of magnitude smaller than the one observed in ordinary CDW materials $(\delta n \ll \delta \Delta)$.

Our method can be further extended to include the effects of magnetic fields by considering a Hubbard model with complex hopping elements (Peierls substitution). In type-II superconductors, external magnetic fields generate isolated vortices in whose core the pairing gap is locally suppressed. Hence, a magnetic vortex acts as a pinning site for a PDW modulation, in analogy to the bond impurity considered in this Rapid Communication. Numerical studies of the Hubbard model in the presence of magnetic fields have indeed found that spatial modulations of the pairing gap develop in the proximity of the vortex core $[70,76,77]$. This finding is consistent with STS experiments demonstrating that the periodic modulations are mostly pronounced in the vicinity of the vortex core $[7,41,78,79]$, as well as with evidence that the density waves become long ranged at high magnetic fields $[2,3,6,22]$. By locally suppressing superconductivity, large densities of magnetic vortices can lead to a long-ranged PDW order.

Acknowledgments. We thank Peter Abbamonte, Lucio Braicovich, Debanjan Chowdhury, Riccardo Comin, J. C. Seamus Davis, Andrea Damascelli, Eugene Demler, Giacomo Ghiringhelli, Marco Grilli, Jenny Hoffman, Amit Keren, Steve Kivelson, and Dror Orgad for useful discussions. This work is supported by the Israel Science Foundation Grants No. $151 / 19$ and No. 967/19. 
[1] J. M. Tranquada, B. J. Sternlieb, J. D. Axe, Y. Nakamura, and S. Uchida, Evidence for stripe correlations of spins and holes in copper oxide superconductors, Nature (London) 375, 561 (1995).

[2] N. Doiron-Leyraud et al., Quantum oscillations and the Fermi surface in an underdoped high- $T_{c}$ superconductor, Nature (London) 447, 565 (2007).

[3] T. Wu et al., Magnetic-field-induced charge-stripe order in the high-temperature superconductor $\mathrm{YBa}_{2} \mathrm{Cu}_{3} \mathrm{O}_{y}$, Nature (London) 477, 191 (2011).

[4] E. Fradkin and S. A. Kivelson, High-temperature superconductivity: Ineluctable complexity, Nat. Phys. 8, 864 (2012).

[5] D. LeBoeuf et al., Thermodynamic phase diagram of static charge order in underdoped $\mathrm{YBa}_{2} \mathrm{Cu}_{3} \mathrm{O}_{y}$, Nat. Phys. 9, 79 (2013).

[6] T. Wu et al., Emergence of charge order from the vortex state of a high-temperature superconductor, Nat. Commun. 4, 2113 (2013).

[7] J. E. Hoffman et al., A four unit cell periodic pattern of quasiparticle states surrounding vortex cores in $\mathrm{Bi}_{2} \mathrm{Sr}_{2} \mathrm{CaCu}_{2} \mathrm{O}_{8+\delta}$, Science 295, 466 (2002).

[8] C. Howald, H. Eisaki, N. Kaneko, M. Greven, and A. Kapitulnik, Periodic density-of-states modulations in superconducting $\mathrm{Bi}_{2} \mathrm{Sr}_{2} \mathrm{CaCu}_{2} \mathrm{O}_{8+\delta}$, Phys. Rev. B 67, 014533 (2003).

[9] M. Vershinin et al., Local ordering in the pseudogap state of the high- $T_{c}$ superconductor $\mathrm{Bi}_{2} \mathrm{Sr}_{2} \mathrm{CaCu}_{2} \mathrm{O}_{8+\delta}$, Science 303, 1995 (2004).

[10] T. Hanaguri et al., A checkerboard electronic crystal state in lightly hole-doped $\mathrm{Ca}_{2-x} \mathrm{Na}_{x} \mathrm{CuO}_{2} \mathrm{Cl}_{2}$, Nature (London) 430, 1001 (2004).

[11] G. Ghiringhelli et al., Long-range incommensurate charge fluctuations in (Y, Nd) $\mathrm{Ba}_{2} \mathrm{Cu}_{3} \mathrm{O}_{6+x}$, Science 337, 821 (2012).

[12] J. Chang et al., Direct observation of competition between superconductivity and charge density wave order in $\mathrm{YBa}_{2} \mathrm{Cu}_{3} \mathrm{O}_{6.67}$, Nat. Phys. 8, 871 (2012).

[13] D. H. Torchinsky, F. Mahmood, A. T. Bollinger, I. Božović, and N. Gedik, Fluctuating charge-density waves in a cuprate superconductor, Nat. Mater. 12, 387 (2013).

[14] E. Blackburn et al., X-Ray Diffraction Observations of a Charge-Density-Wave Order in Superconducting Ortho-II $\mathrm{YBa}_{2} \mathrm{Cu}_{3} \mathrm{O}_{6.54}$ Single Crystals in Zero Magnetic Field, Phys. Rev. Lett. 110, 137004 (2013).

[15] E. Comin et al., Charge order driven by Fermi-arc instability in $\mathrm{Bi}_{2} \mathrm{Sr}_{2-x} \mathrm{La}_{x} \mathrm{CuO}_{6+\delta}$, Science 343, 390 (2014).

[16] E. H. da Silva Neto et al., Ubiquitous interplay between charge ordering and high-temperature superconductivity in cuprates, Science 343, 393 (2014).

[17] M. Le Tacon et al., Inelastic x-ray scattering in $\mathrm{YBa}_{2} \mathrm{Cu}_{3} \mathrm{O}_{6.6}$ reveals giant phonon anomalies and elastic central peak due to charge-density-wave formation, Nat. Phys. 10, 52 (2014).

[18] M. Hashimoto, G. Ghiringhelli, W. S. Lee, G. Dellea, A. Amorese, C. Mazzoli, K. Kummer, N. B. Brookes, B. Moritz, Y. Yoshida et al., Direct observation of bulk charge modulations in optimally doped $\mathrm{Bi}_{1.5} \mathrm{~Pb}_{0.6} \mathrm{Sr}_{1.54} \mathrm{CaCu}_{2} \mathrm{O}_{8+\delta}$, Phys. Rev. B 89, 220511(R) (2014).

[19] W. Tabis et al., Charge order and its connection with Fermiliquid charge transport in a pristine high- $T_{c}$ cuprate, Nat. Commun. 5, 5875 (2014).
[20] M. Huecker et al., Competing charge, spin, and superconducting orders in underdoped $\mathrm{YBa}_{2} \mathrm{Cu}_{3} \mathrm{O}_{y}$, Phys. Rev. B 90, 054514 (2014).

[21] A. J. Achkar, X. Mao, C. McMahon, R. Sutarto, F. He, R. Liang, D. A. Bonn, W. N. Hardy, and D. G. Hawthorn, Impact of Quenched Oxygen Disorder on Charge Density Wave Order in $\mathrm{YBa}_{2} \mathrm{Cu}_{3} \mathrm{O}_{6+x}$, Phys. Rev. Lett. 113, 107002 (2014).

[22] S. Gerber et al., Three-dimensional charge density wave order in $\mathrm{YBa}_{2} \mathrm{Cu}_{3} \mathrm{O}_{6.67}$ at high magnetic fields, Science 350, 949 (2015).

[23] M. H. Hamidian et al., Magnetic-field induced interconversion of Cooper pairs and density wave states within cuprate composite order, arXiv:1508.00620.

[24] Y. Y. Peng et al., Direct observation of charge order in underdoped and optimally doped $\mathrm{Bi}_{2}(\mathrm{Sr}, \mathrm{La})_{2} \mathrm{CuO}_{6+\delta}$ by resonant inelastic x-ray scattering, Phys. Rev. B 94, 184511 (2016).

[25] L. Chaix et al., Dispersive charge density wave excitations in $\mathrm{Bi}_{2} \mathrm{Sr}_{2} \mathrm{CaCu}_{2} \mathrm{O}_{8+\delta}$, Nat. Phys. 13, 952 (2017).

[26] Y. Y. Peng et al., Re-entrant charge order in overdoped $(\mathrm{Bi}, \mathrm{Pb})_{2.12} \mathrm{Sr}_{1.88} \mathrm{CuO}_{6+\delta}$ outside the pseudogap regime, Nat. Mater. 17, 697 (2018).

[27] H. Jang, S. Asano, M. Fujita, M. Hashimoto, D. H. Lu, C. A. Burns, C. C. Kao, and J. S. Lee, Superconductivity-Insensitive Order at $q \sim 1 / 4$ in Electron-Doped Cuprates, Phys. Rev. X 7 , 041066 (2017).

[28] E. H. da Silva Neto, M. Minola, B. Yu, W. Tabis, M. Bluschke, D. Unruh, H. Suzuki, Y. Li, G. Yu, D. Betto et al., Coupling between dynamic magnetic and charge-order correlations in the cuprate superconductor $\mathrm{Nd}_{2-x} \mathrm{Ce}_{x} \mathrm{CuO}_{4}$, Phys. Rev. B 98 161114(R) (2018).

[29] M. Bluschke, M. Yaari, E. Schierle, G. Bazalitsky, J. Werner, E. Weschke, and A. Keren, Adiabatic variation of the charge-density-wave phase diagram in the 123 cuprate $\left(\mathrm{Ca}_{x} \mathrm{La}_{1-x}\right)\left(\mathrm{Ba}_{1.75-x} \mathrm{La}_{0.25+x}\right) \mathrm{Cu}_{3} \mathrm{O}_{y}$, Phys. Rev. B 100, 035129 (2019).

[30] M. Kang et al., Evolution of charge order topology across a magnetic phase transition in cuprate superconductors, Nat. Phys. 15, 335 (2019).

[31] C. Castellani, C. Di Castro, and M. Grilli, Singular Quasiparticle Scattering in the Proximity of Charge Instabilities, Phys. Rev. Lett. 75, 4650 (1995).

[32] C. Castellani, C. Di Castro, and M. Grilli, The charge-densitywave quantum-critical-point scenario, Phys. C (Amsterdam, Neth.) 282, 260 (1997).

[33] R. H. He et al., From a single-band metal to a high-temperature superconductor via two thermal phase transitions, Science 331, 1579 (2011).

[34] B. Loret et al., Intimate link between charge density wave, pseudogap and superconducting energy scales in cuprates, Nat. Phys. 15, 771 (2019).

[35] H. D. Chen, O. Vafek, A. Yazdani, and S. C. Zhang, Pair Density Wave in the Pseudogap State of High Temperature Superconductors, Phys. Rev. Lett. 93, 187002 (2004).

[36] P. A. Lee, Amperean Pairing and the Pseudogap Phase of Cuprate Superconductors, Phys. Rev. X 4, 031017 (2014).

[37] C. Pépin, V. S. De Carvalho, T. Kloss, and X. Montiel, Pseudogap, charge order, and pairing density wave at the hot spots in cuprate superconductors, Phys. Rev. B 90, 195207 (2014). 
[38] H. Freire, V. S. De Carvalho, and C. Pépin, Renormalization group analysis of the pair-density-wave and charge order within the fermionic hot-spot model for cuprate superconductors, Phys. Rev. B 92, 045132 (2015).

[39] Y. Wang, D. F. Agterberg, and A. Chubukov, Interplay between pair-and charge-density-wave orders in underdoped cuprates, Phys. Rev. B 91, 115103 (2015).

[40] Y. Wang, D. F. Agterberg, and A. Chubukov, Coexistence of Charge-Density-Wave and Pair-Density-Wave Orders in Underdoped Cuprates, Phys. Rev. Lett. 114, 197001 (2015).

[41] S. D. Edkins et al., Magnetic field-induced pair density wave state in the cuprate vortex halo, Science 364, 976 (2019).

[42] M. H. Hamidian et al., Detection of a Cooper-pair density wave in $\mathrm{Bi}_{2} \mathrm{Sr}_{2} \mathrm{CaCu}_{2} \mathrm{O}_{8+x}$, Nature (London) 532, 343 (2016).

[43] Z. Du et al., Imaging the energy gap modulations of the cuprate pair-density-wave state, Nature (London) 580, 65 (2020).

[44] D. F. Agterberg et al., The physics of pair density waves, Annu. Rev. Condens. Matter Phys. 11, 231 (2020).

[45] E. G. Dalla Torre, D. Benjamin, Y. He, D. Dentelski, and E. Demler, Friedel oscillations as a probe of fermionic quasiparticles, Phys. Rev. B 93, 205117 (2016).

[46] R. Arpaia et al., Dynamical charge density fluctuations pervading the phase diagram of a Cu-based high- $T_{c}$ superconductor, Science 365, 906 (2019).

[47] S. Sachdev and R. La Placa, Bond Order in Two-Dimensional Metals with Antiferromagnetic Exchange Interactions, Phys. Rev. Lett. 111, 027202 (2013).

[48] K. B. Efetov, H. Meier, and C. Pépin, Pseudogap State Near a Quantum Critical Point, Nat. Phys. 9, 442 (2013).

[49] A. Allais, J. Bauer, and S. Sachdev, Density wave instabilities in a correlated two-dimensional metal, Phys. Rev. B 90, 155114 (2014).

[50] Y. Caplan, G. Wachtel, and D. Orgad, Long-range order and pinning of charge-density waves in competition with superconductivity, Phys. Rev. B 92, 224504 (2015).

[51] Y. Caplan and D. Orgad, Dimensional Crossover of ChargeDensity Wave Correlations in the Cuprates, Phys. Rev. Lett. 119, 107002 (2017).

[52] A. Altland and B. D. Simons, Condensed Matter Field Theory (Cambridge University Press, Cambridge, UK, 2010).

[53] According to the interpretation of the pseudogap as a precursor of the superconducting pairing gap [80], this approach applies to the pseudogap region as well.

[54] S. Massidda, N. Hamada, J. Yu, and A. J. Freeman, Electronic structure of Nd-Ce-Cu-O, a Fermi liquid superconductor, Phys. C (Amsterdam, Neth.) 157, 571 (1989).

[55] See Supplemental Material at http://link.aps.org/supplemental/ 10.1103/PhysRevResearch.2.032012 for the doping dependence of our model, compared to the experimental findings. Increasing the hole doping reduces the antinodal distance and, accordingly, reduces the CDW/PDW wave vector observed in BSCCO [81,15] and YBCO [14,20]. Some authors [15,27] disputed this interpretation due to the mismatch between the wave vector observed in x-ray experiments and the antinodal distance observed in ARPES. We point out that this mismatch is rather small (of the order of 10\%) and is of the same order as the mismatch between the antinodal distances obtained by fitting the ARPES data with different phenomenological band structures $[82,15]$. Furthermore, x-ray scattering is sensitive to the bulk of the material, while ARPES is a surface probe. Hence, the mismatch between these two probes may be due to the common bulk-surface dichotomy of the chemical potential.

[56] Note that the integrals in Eq. (3) involve diverging functions. To normalize these expressions, we substituted $\Omega \rightarrow \Omega+i \Gamma$ with $\Gamma / t=0.1$ and summed over $N=301$ equally spaced points (see the Supplemental Material). We checked that the qualitative features of the resulting plots do not depend on the normalization procedure.

[57] M. R. Norman, M. Randeria, H. Ding, and J. C. Campuzano, Phenomenological models for the gap anisotropy of $\mathrm{Bi}_{2} \mathrm{Sr}_{2} \mathrm{CaCu}_{2} \mathrm{O}_{8}$ as measured by angle-resolved photoemission spectroscopy, Phys. Rev. B 52, 615 (1995).

[58] R. S. Markiewicz, S. Sahrakorpi, M. Lindroos, H. Lin, and A. Bansil, One-band tight-binding model parametrization of the high- $T_{c}$ cuprates including the effect of $k_{z}$ dispersion, Phys. Rev. B 72, 054519 (2005).

[59] P. Fulde and R. A. Ferrell, Superconductivity in a strong spinexchange field, Phys. Rev. 135, A550 (1964).

[60] A. I. Larkin and Y. N. Ovchinnikov, Nonuniform state of superconductors, Zh. Eksp. Teor. Fiz. 47, 1136 (1964) [Sov. Phys.-JETP 20, 762 (1965)].

[61] E. Berg, E. Fradkin, and S. A. Kivelson, Theory of the striped superconductor, Phys. Rev. B 79, 064515 (2009).

[62] E. Berg, E. Fradkin, S. A. Kivelson, and J. M. Tranquada, Striped superconductors: How spin, charge and superconducting orders intertwine in the cuprates, New J. Phys. 11, 115004 (2009).

[63] See also the Supplemental Material for a simplified model of the Fermi surface of cuprates predicting that for CDWs with $q / 2 \pi=0.25, R=3 / 4$.

[64] G. Ghiringhelli (private communication).

[65] R. Micnas, J. Ranninger, and S. Robaszkiewicz, An extended Hubbard model with inter-site attraction in two dimensions and high- $T_{c}$ superconductivity, J. Phys. C 21, L145 (1988).

[66] R. Micnas, J. Ranninger, and S. Robaszkiewicz, "Superconductivity in narrow-band systems with local nonretarded attractive interactions," Rev. Mod. Phys. 62, 113 (1990).

[67] P. H. Monthoux and D. J. Scalapino, Self-Consistent $d_{x^{2}-y^{2}}$ Pairing in a Two-Dimensional Hubbard Model, Phys. Rev. Lett. 72, 1874 (1994).

[68] D. M. Newns, C. C. Tsuei, and P. C. Pattnaik, Van Hove scenario for $d$-wave superconductivity in cuprates, Phys. Rev. B 52, 13611 (1995).

[69] T. Husslein, I. Morgenstern, D. M. Newns, P. C. Pattnaik, J. M. Singer, and H. G. Matuttis, Quantum Monte Carlo Evidence for $d$-wave pairing in the two-dimensional Hubbard model at a van Hove singularity, Phys. Rev. B 54, 16179 (1996).

[70] M. Takigawa, M. Ichioka, and K. Machida, Quasiparticle structure in antiferromagnetism around the vortex and nuclear magnetic relaxation time, J. Phys. Soc. Jpn. 73, 450 (2004).

[71] The relative standard deviation of $X$ is defined as $\delta X \equiv$ $\left(\Sigma_{j} X_{j}^{2}\right)^{1 / 2} / \Sigma_{j} X_{j}$.

[72] T. Pereg-Barnea and M. Franz, Theory of quasiparticle interference patterns in the pseudogap phase of the cuprate superconductors, Phys. Rev. B 68, 180506 (2003).

[73] E. A. Nowadnick, B. Moritz, and T. P. Devereaux, Quasiparticle interference and the interplay between superconductivity and 
density wave order in the cuprates, Phys. Rev. B 86, 134509 (2012).

[74] E. G. Dalla Torre, Y. He, D. Benjamin, and E. Demler, Exploring quasiparticles in high- $T_{c}$ cuprates through photoemission, tunneling, and x-ray scattering experiments, New J. Phys. 17, 022001 (2015).

[75] Z. Dai, Y. H. Zhang, T. Senthil, and P. A. Lee, Pair-density waves, charge-density waves, and vortices in high- $T_{c}$ cuprates, Phys. Rev. B 97, 174511 (2018).

[76] J. X. Zhu, I. Martin, and A. R. Bishop, Spin and Charge Order Around Vortices and Impurities in High- $T_{c}$ Superconductors, Phys. Rev. Lett. 89, 067003 (2002).

[77] S. Simonucci, P. Pieri, and G. C. Strinati, Temperature dependence of a vortex in a superfluid Fermi gas, Phys. Rev. B 87, 214507 (2013).
[78] K. Matsuba et al., Anti-phase modulation of electron- and holelike states in vortex core of $\mathrm{Bi}_{2} \mathrm{Sr}_{2} \mathrm{CaCu}_{2} \mathrm{O}_{x}$ probed by scanning tunneling spectroscopy, J. Phys. Soc. Jpn. 76, 063704 (2007).

[79] S. Yoshizawa et al., High-resolution scanning tunneling spectroscopy of vortex cores in inhomogeneous electronic states of $\mathrm{Bi}_{2} \mathrm{Sr}_{2} \mathrm{CaCu}_{2} \mathrm{O}_{x}$, J. Phys. Soc. Jpn. 82, 083706 (2013).

[80] V. J. Emery and S. A. Kivelson, Importance of phase fluctuations in superconductors with small superfluid density, Nature (London) 374, 434 (1995).

[81] W. D. Wise et al., Charge-density-wave origin of cuprate checkerboard visualized by scanning tunneling microscopy, Nat. Phys. 4, 696 (2008).

[82] P. D. C. King et al., Structural Origin of Apparent Fermi Surface Pockets in Angle-Resolved Photoemission of $\mathrm{Bi}_{2} \mathrm{Sr}_{2-x} \mathrm{La}_{x} \mathrm{CuO}_{6+\delta}$, Phys. Rev. Lett. 106, 127005 (2011). 